\title{
An Analysis for Aggravation of Thyroid Function After Discontinuing Potassium lodine in Graves' Patients Treated With Methimazole and Potassium lodine
}

\author{
Seigo Tachibana ${ }^{\mathrm{a}, \mathrm{b}, \mathrm{d}}$, Yusuke Moric ${ }^{\mathrm{c}}$, Tadao Yokoi $^{\mathrm{c}}$, Shinya Sato ${ }^{\mathrm{c}}$, \\ Toshihiko Yanase ${ }^{b}$, Hiroyuki Yamashita ${ }^{c}$
}

\begin{abstract}
Background: The efficacy of methimazole (MMI) combined with potassium iodine (KI) therapy as an initial treatment of Graves' disease (GD) is reported in an area of excessive iodine intake. However, transient aggravation of thyroid function after cessation of KI is often observed. Therefore, we evaluated the factors related with transient aggravation after cessation of KI in GD patients treated with MMI and KI therapy.
\end{abstract}

Methods: This was a retrospective chart review. Seventy-three GD patients were enrolled in this study. Subjects were divided into two groups. Subjects with transient aggravation were group A, and those without transient aggravation were group B. Between two groups, possible factors were evaluated. Standardized assays were used to measure TSH, free T4 (FT4), free T3 (FT3) and thyrotropinbinding inhibitor immunoglobulin (TRAb). Ultrasonography was used to estimate thyroid volume.

Results: Fisher's exact test or Mann-Whitney U test revealed that "firstly adjusted drugs", "the duration of FT4 normalization from initiation of medication to correction of FT4 to normal range" and "adoption of simultaneous reduction of both MMI and KI" are significant factors. In addition, the latter two factors stayed significant even by multiple logistic regression analysis.

Conclusion: Our study suggested that the methods of drugs' adjustment and "the duration from initiation of medication to normal-

\footnotetext{
Manuscript accepted for publication December 27, 2013

${ }^{a}$ Department of Endocrinology, Yamashita Thyroid and Parathyroid Clinic, 1-8 Shimogofukumachi, Hakata-ku, Fukuoka-city 812-0034, Japan

${ }^{b}$ Department of Endocrinology and Diabetes Medicine, Fukuoka University Hospital, 7-45-1 Nanakuma, Jonan-ku, Fukuoka-city 814-0180, Japan

${ }^{\mathrm{c}}$ Department of Surgery, Yamashita Thyroid and Parathyroid Clinic, 1-8 Shimogofukumachi, Hakata-ku, Fukuoka-city 812-0034, Japan

${ }^{\mathrm{d}}$ Corresponding author: Seigo Tachibana, 1-8 Shimogofukumachi, Hakata-ku, Fukuoka-city 812-0034, Japan.

Email: Tachibana@kojosen.com
}

doi: http://dx.doi.org/10.4021/jem204w ization of thyroid hormone" may be significant factors of transient aggravation after cessation of KI. It may be better not to reduce both drugs simultaneously, especially in patients who show the relatively shorter duration of normalization of thyroid function.

Keyword: Graves' disease; Methimazole; Potassium iodine; Transient aggravation

\section{Introduction}

The first-line therapy for Graves' disease (GD) is antithyroid drug (ATD) therapy in many countries $[1,2]$. However, the various adverse effects are often observed. In particular, agranulocytosis is the most annoying problem because this adverse effect is often life-threatening. Agranulocytosis induced by methimazole (MMI) is well known as the dose-dependent adverse effect [3]. In fact, the frequency of agranulocytosis by MMI at a dose of $15 \mathrm{mg}$ per day was significantly lower than that by MMI at a dose of $30 \mathrm{mg}$ per day. Thus, one way to reduce the risk of agranulocytosis by MMI is the combination therapy of a relatively small dose of MMI and potassium iodine (KI) in the initiation therapy. Actually, the administration of KI for GD patients has been shown to be one useful therapeutic choice, which can correct thyroid function very rapidly. It is well known that adequate dose of KI suppresses the release and synthesis of thyroid hormone in the thyroid of GD patients [4, 5]. In situations that a rapid correction of thyroid function is required, such as severe thyrotoxicosis containing thyroid storm and preparation for urgent thyroidectomy, KI treatment has also been reported to be definitely advantageous [6-9].

Under these backgrounds, Takata et al reported that initial therapy using MMI combined with KI is useful to correct thyroid function in GD patients in Japan [10]. In their report, when free T4 (FT4) was corrected to the normal range, KI was discontinued. After cessation of KI, transient aggravation of thyroid function was observed in $8.1-9.4 \%$ of patients [10]. However, little is known about the factors associated with the aggravation after cessation of KI. It will be clinically meaningful if we could predict who are able to continue 
Table 1. Profile of Subjects (Mean \pm SD)

$\begin{array}{ll}\text { Male:female } & 13: 60 \\ \text { Age } & 14-67 \text { (median 37) } \\ \text { FT4 before treatment } & 5.52 \pm 1.73 \mathrm{ng} / \mathrm{dL} \\ \text { FT3 before treatment } & 19.50 \pm 6.97 \mathrm{pg} / \mathrm{mL} \\ \text { Thyroid volume before treatment } & 27.1 \pm 12.5 \mathrm{~g} \\ \text { TRAb before treatment } & 16.0 \pm 7.8 \mathrm{IU} / \mathrm{L}\end{array}$

or discontinue the KI treatment under the combined therapy with MMI.

In the present study, we evaluated the factors related with transient aggravation of thyroid function after cessation of KI in GD patients treated with MMI and KI therapy.

\section{Materials and Methods}

\section{Subjects}

Between April 2010 and December 2012, 565 GD patients visited Yamashita Thyroid and Parathyroid Clinic. Of these, 87 patients received MMI combined with KI therapy as an initial treatment, because their concentrations of thyroid hormone were extremely high, or their clinical manifestations associated with thyrotoxicosis were remarkable. Five patients who suffered from adverse effects of MMI were excluded. Nine patients who had to increase MMI or KI before first reduction of drugs were also excluded from this series. Therefore, 73 patients who could be observed at least until thyroid function was corrected to normal range were enrolled in this study. The clinical and biochemical profile of subjects is shown in Table 1. The diagnosis of GD was based on hyperthyroidism (suppressed serum TSH level and elevated levels of serum free T3 (FT3) and FT4), thyrotropin-binding inhibitor immunoglobulin (TRAb) positive and/ or increased radioiodine uptake.

\section{Treatment}

Both MMI (daily dose of $10-20 \mathrm{mg}$ ) and KI (daily dose of $50 \mathrm{mg}$ ) were prescribed to these subjects. The dose of MMI was decided by each two doctors' judgment based on concentrations of thyroid hormones and thyroid volume. When FT4 was corrected to normal range, KI or/and MMI were reduced. The dose adjustment of MMI or KI was performed by each doctor's decision based on the results of medical examination. When the KI dose was judged to be proper, 50 $\mathrm{mg}$ of KI medication was continued for a while and stopped or tapered. In the tapering of $\mathrm{KI}$, the dose was reduced to 25 mg per day, continued for a while and finally stopped. MMI was tapered every $5 \mathrm{mg}$ at the reduction of MMI.

\section{Laboratory measurements}

TSH, FT3, FT4 and TRAb were examined using ECLusys kit (Roche Diagnostics, Penzberg, Germany). The reference ranges were as follows: TSH $0.5-5.0 \mathrm{mIU} / \mathrm{L}, \mathrm{FT} 32.3-4.3$ $\mathrm{pg} / \mathrm{mL}$ and FT4 0.9 - $1.7 \mathrm{ng} / \mathrm{dL}$. TRAb measurement was performed using anti-M22 antibody called third generation (ECLusys kit; Roche Diagnostics, Penzberg, Germany). The reference range of TRAb was below 2.0 IU/L. During the follow-up period, measurement of TSH and FT4 was performed every 2 - 4 weeks.

\section{Study Protocol}

This was a retrospective chart review. Seventy-three patients were divided into the following two groups. Thirty-one patients (26 male and 5 female) who showed transient aggravation of thyroid function after cessation of KI were categorized group $\mathrm{A}$, while 42 patients ( 34 male and 8 female) who did not show such aggravation were categorized group B. The transient aggravation of thyroid function was defined by elevation of serum FT4 and/or FT3 concentration over normal range 2 - 4 weeks after cessation of KI. Between these two groups, we evaluated following factors: gender, age, thyroid function (FT4, FT3 and TRAb) and thyroid volume before treatment, initial dose of MMI, firstly adjusted drugs (MMI or KI), FT4 at first adjustment of drugs, the duration from initiation of medication to correction of FT4 to normal range, the duration of administration of KI, methods of adjustment of KI (tapering or cessation of $50 \mathrm{mg}$ KI without tapering) and adoption of simultaneous reduction of both MMI and KI. The volume of each thyroid lobe was calculated by following formula: $\pi / 6 \times$ width $\times$ length $\times$ depth. The thyroid volume was calculated by sum of the volume of each thyroid lobe. In this series, the simultaneous adjustment of both MMI and KI at the first drug adjustment was not included. Therefore, the first adjustment of drugs was performed by only one drug, and the simultaneous reduction of both MMI 
Table 2. Results of Statistical Analysis (Mean \pm SD)

\begin{tabular}{|c|c|c|c|}
\hline & Group A & Group B & P value \\
\hline Gender (male:female) & $26: 5$ & $34: 8$ & 1.00 \\
\hline Age & $35.8 \pm 11.9$ & $39.0 \pm 12.2$ & 0.23 \\
\hline FT4 before treatment $(\mathrm{ng} / \mathrm{dL})$ & $5.63 \pm 1.72$ & $5.40 \pm 1.74$ & 0.64 \\
\hline FT3 before treatment $(\mathrm{pg} / \mathrm{mL})$ & $20.33 \pm 7.14$ & $18.90 \pm 6.88$ & 0.48 \\
\hline TRAb before treatment (IU/L) & $16.73 \pm 8.46$ & $15.39 \pm 7.41$ & 0.53 \\
\hline Thyroid volume before treatment (g) & $30.13 \pm 14.64$ & $24.85 \pm 10.19$ & 0.12 \\
\hline Initial dose of MMI (mg) & $14.84 \pm 1.57$ & $14.17 \pm 1.89$ & 0.12 \\
\hline Initially adjusted drugs (MMI:KI) & $11: 20$ & $4: 38$ & $0.009^{*}$ \\
\hline FT4 at first adjustment of drugs (ng/dL) & $1.36 \pm 0.30$ & $1.25 \pm 0.38$ & 0.09 \\
\hline $\begin{array}{l}\text { The duration from initiation of medication to correction } \\
\text { of FT4 to normal range (days) }\end{array}$ & $31.2 \pm 18.3$ & $53.9 \pm 40.6$ & $0.002 *$ \\
\hline The duration of administration of KI (days) & $55.2 \pm 36.9$ & $72.4 \pm 56.6$ & 0.15 \\
\hline $\begin{array}{l}\text { Methods of adjustment of KI (tapering:cessation without } \\
\text { tapering) }\end{array}$ & $16: 15$ & $13: 29$ & 0.09 \\
\hline $\begin{array}{l}\text { Adoption of simultaneous reductions of both MMI and } \\
\text { KI (yes:no) }\end{array}$ & $10: 21$ & $3: 39$ & $0.011^{*}$ \\
\hline
\end{tabular}

All factors were evaluated by Fisher's exact test or Mann-Whitney U test. "Initially adjusted drugs", "the duration from initiation of medication to correction of FT4 to normal range" and "adoption of simultaneous reductions of both MMI and Kl" showed significant differences between these groups.

and KI was performed from the second drug adjustment. In addition, these subjects were continued to follow for at least 3 months after cessation of KI.

\section{Statistical analysis}

In these two groups, statistical analysis was performed with Fisher's exact test and Mann-Whitney U test. In addition, multiple logistic regression analysis was performed with factors showing significant differences by Fisher's exact test and Mann-Whitney U test. These analyses were performed by JMP ver.6.0 (SAS Institute Inc.).

\section{Results}

As shown in Table 2, as to gender, age, FT4, FT3, TRAb, thyroid volume before treatment, initial dose of MMI, FT4 at first adjustment of drugs and the duration of administration of KI, no statistical difference was observed between groups A and B. However, "firstly adjusted drugs", "the du- ration from initiation of medication to correction of FT4 to normal range" and "adoption of simultaneous reduction of both MMI and KI" showed significant differences between these groups by Fisher's exact test or Mann-Whitney U test (Table 2) $(\mathrm{P}=0.009, \mathrm{P}=0.005, \mathrm{P}=0.011$, respectively). The reduction of MMI at the first adjustment of drugs and the relatively shorter duration from initiation of medication to correction of FT4 tended to show transient aggravation of thyroid function after cessation of KI (31.2 \pm 18.3 vs. 53.9 \pm 40.6 days). In addition, patients with simultaneous reduction of both MMI and KI were more likely to show transient exaggeration of thyroid function.

Using above-mentioned three significant factors, multiple logistic regression analysis was performed. "The duration from initiation of medication to correction of FT4 to normal range" and "adoption of simultaneous reduction" significantly contributed to transient aggravation of thyroid function after cessation of $\mathrm{KI}(\mathrm{P}=0.03, \mathrm{P}=0.03, \mathrm{CI} 95 \%)$. However, contribution ratio of this model was $21 \%$. Odds ratio of "the duration from initiation of medication to correction of FT4" was 0.97 , and that of "adoption of simultaneous 
Table 3. Results of Multiple Logistic Regression Analysis

\begin{tabular}{llll}
\hline & Odds ratio & CI 95\% & P value \\
\hline $\begin{array}{l}\text { Initially adjusted drugs } \\
\begin{array}{l}\text { The duration from initiation of medication to correction } \\
\text { of FT4 to normal range }\end{array}\end{array}$ & 0.97 & $0.93-3.79$ & 0.09 \\
$\begin{array}{l}\text { Adoption of simultaneous reductions of both MMI and } \\
\text { KI }\end{array}$ & 2.39 & $1.16-5.73$ & $0.02 *$ \\
\hline
\end{tabular}

Contribution 21\%. "The duration from initiation of medication to correction of FT4 to normal range" and "adoption of simultaneous reductions" significantly contributed to transient aggravation of thyroid function after the cessation of KI by multiple logistic regression analysis. However, the proportion contributed by this model was $21 \%$.

reduction" was 2.39 as shown in Table 3.

\section{Discussion}

$\mathrm{KI}$ is often prescribed to patients with severe thyrotoxicosis because it can effectively suppress thyroid hormone release $[5,7,11]$. However, it is also well known that this effect sometimes becomes ineffective during the treatment. Although almost all patients respond initially, about one-third respond only partially and remain toxic, and another onethird respond initially but relapse after about 6 weeks [12]. Therefore, KI has been generally prescribed in limited situations such as preparation for thyroidectomy, treatment after radioactive iodine therapy for GD and correction of thyroid function of patients suffering from adverse effects of ATDs $[6,7,13,14]$. In addition, iodine administration in combination with ATDs has been reported to reduce the efficacy of ATDs in vitro [15-17]. There are also some reports that the combination of ATDs with iodine was not effective and KI monotherapy of GD impaired the efficacy of subsequent ATDs therapy $[18,19]$. Moreover, it is reported that MMI more effectively reduces thyroid hormone in GD patients living in countries with a moderately low iodine intake, compared with that in those with sufficient dietary iodine intake [20]. Considering these backgrounds, in many countries, MMI combined with KI therapy is seldom selected as the first-line therapy in GD patients. However, in Japan that is one of countries with sufficient dietary iodine intake, Takata et al reported the efficacy of MMI combined with KI as for the initial therapy in GD patients as far as reduction of a side effect of ATDs and early hormonal normalization [10]. In addition, Kasai et al reported the efficacy of propylthiouracil combined with small dose of iodine in the early period of GD treatment in Japan [21], and Hiraiwa et al reported that restriction of dietary iodine dose not ameliorate the early effect of ATD for GD patients in Japan [22]. Therefore, in Japan,
MMI combined with KI therapy has recently become one of significant options for the initial treatments in GD patients. However, the protocol of efficient drug reduction without the transient aggravation of thyroid function remains to be established due to the lack of information for its risk factors. In this respect, we evaluated factors in relation with the transient aggravation of thyroid function after cessation of KI.

Interestingly, Fisher's exact test or Mann-Whitney U test revealed that "firstly adjusted drugs", "the duration of FT4 normalization from initiation of medication to correction of FT4 to normal range" and "adoption of simultaneous reduction of both MMI and KI" are significant factors to differentiate groups with (A) and without (B) aggravation of thyroid function after cessation of KI. In addition, the latter two factors stayed significant even by multiple logistic regression analysis. Usually, it is very difficult to estimate the extent of KI contribution to the correction of thyroid function at the adjustment of drugs. Therefore, the overreduction of MMI in the early period of GD treatment might happen in the adjustment process of MMI and KI, thus leading to the difficulty to maintain euthyroidism. This may be the possible speculation for the reason that "simultaneous reduction of both MMI and KI" was left as a contribution factor to the aggravation of thyroid function after cessation of KI.

Another interesting point in our statistical finding was that the shorter the duration of FT4 normalization from initiation of medication was, the aggravation of the thyroid function after cessation of KI tended to occur. In a previous study, the group treated with MMI and KI showed more significant improvement of thyroid function than the group with only MMI at $2-4$ weeks after the therapy. From 8 weeks downward, this significant improvement was not observed [10]. These results suggest that KI contributed to normalization of thyroid function at the early period of GD treatment. In other words, a role of KI for FT4 normalization is limited and relatively short. So, collecting together our finding and the previous finding [10], the cessation of KI or tapering of 
MMI should be carefully performed especially in subjects who show the short duration from initiation of medication to FT4 normalization.

There is a report that excess iodine increased thyroid volume of GD patient [23]. Iodine, also known as an immune stimulant, may precipitate the onset of autoimmune thyroid disease and may be also directly toxic to the thyroid epithelial cell [24]. Therefore, a long-term administration of $\mathrm{KI}$ may not be preferable. The usage of KI in the treatment of GD is still controversial and has not been fully established. The further study to establish appropriate way for initial treatment using MMI and KI is needed and currently undergoing in our institution.

In conclusion, our study suggested that the methods of drug adjustment and "the duration from initiation of medication to normalization of thyroid hormone" may be significant factors of transient aggravation after cessation of $\mathrm{KI}$ in patients with GD treated with MMI combined with KI. It may be better not to reduce both drugs simultaneously, especially in patients who show the relatively shorter duration of normalization of thyroid function.

\section{Disclosure}

None of the authors have any potential conflicts of interest associated with this research.

\section{References}

1. Wartofsky L, Glinoer D, Solomon B, Nagataki S, Lagasse R, Nagayama Y, Izumi M. Differences and similarities in the diagnosis and treatment of Graves' disease in Europe, Japan, and the United States. Thyroid. 1991;1(2):129-135.

2. Burch HB, Burman KD, Cooper DS. A 2011 survey of clinical practice patterns in the management of Graves' disease. J Clin Endocrinol Metab. 2012;97(12):45494558.

3. Takata K, Kubota S, Fukata S, Kudo T, Nishihara E, Ito M, Amino N, et al. Methimazole-induced agranulocytosis in patients with Graves' disease is more frequent with an initial dose of $30 \mathrm{mg}$ daily than with $15 \mathrm{mg}$ daily. Thyroid. 2009;19(6):559-563.

4. Nagataki S, Shizume K, Nakao K. Effect of iodide on thyroidal iodine turnover in hyperthyroid subjects. J Clin Endocrinol Metab. 1970;30(4):469-478.

5. Wartofsky L, Ransil BJ, Ingbar SH. Inhibition by iodine of the release of thyroxine from the thyroid glands of patients with thyrotoxicosis. J Clin Invest. 1970;49(1):7886.

6. Cooper DS. Hyperthyroidism. Lancet. 2003;362(9382): 459-468.
7. Burch HB, Wartofsky L. Life-threatening thyrotoxicosis. Thyroid storm. Endocrinol Metab Clin North Am. 1993;22(2):263-277.

8. Erbil Y, Ozluk Y, Giris M, Salmaslioglu A, Issever H, Barbaros U, Kapran Y, et al. Effect of lugol solution on thyroid gland blood flow and microvessel density in the patients with Graves' disease. J Clin Endocrinol Metab. 2007;92(6):2182-2189.

9. Feek CM, Sawers JS, Irvine WJ, Beckett GJ, Ratcliffe WA, Toft AD. Combination of potassium iodide and propranolol in preparation of patients with Graves' disease for thyroid surgery. N Engl J Med. 1980;302(16):883885 .

10. Takata K, Amino N, Kubota S, Sasaki I, Nishihara E, Kudo T, Ito M, et al. Benefit of short-term iodide supplementation to antithyroid drug treatment of thyrotoxicosis due to Graves' disease. Clin Endocrinol (Oxf). 2010;72(6):845-850.

11. Woeber KA. Iodine and thyroid disease. Med Clin North Am. 1991;75(1):169-178.

12. Emerson $\mathrm{CH}$, Anderson AJ, Howard WJ, Utiger RD. Serum thyroxine and triiodothyronine concentrations during iodide treatment of hyperthyroidism. J Clin Endocrinol Metab. 1975;40(1):33-36.

13. Ross DS, Daniels GH, De Stefano P, Maloof F, Ridgway EC. Use of adjunctive potassium iodide after radioactive iodine (131I) treatment of Graves' hyperthyroidism. J Clin Endocrinol Metab. 1983;57(2):250-253.

14. Kubota S, Ohye H, Sasaki I, Nishihara E, Kudo T, Fukata S, Amino N, et al. Successful use of iodine and levothyroxine to treat Graves' disease in a pregnant patient with allergy to antithyroid drugs and high thyrotropinbinding inhibitor immunoglobulin after radioiodine therapy. Thyroid. 2005;15(12):1373-1376.

15. Taurog A. The mechanism of action of the thioureylene antithyroid drugs. Endocrinology. 1976;98(4):10311046.

16. Hashizume K, Akasu F, Takazawa K, Endo W, Onaya T. The inhibitory effect of acute administration of excess iodide on the formation of adenosine 3', 5'-monophosphate induced by thyrotropin in mouse thyroid lobes. Endocrinology. 1976;99(6):1463-1468.

17. Davidson B, Soodak M, Neary JT, Strout HV, Kieffer JD, Mover H, Maloof F. The irreversible inactivation of thyroid peroxidase by methylmercaptoimidazole, thiouracil, and propylthiouracil in vitro and its relationship to in vivo findings. Endocrinology. 1978;103(3):871882.

18. Roti E, Robuschi G, Gardini E, Montermini M, Salvi M, Manfredi A, Gnudi A, et al. Comparison of methimazole, methimazole and sodium ipodate, and methimazole and saturated solution of potassium iodide in the early treatment of hyperthyroid Graves' disease. Clin Endocrinol (Oxf). 1988;28(3):305-314. 
19. Martino E, Balzano S, Bartalena L, Loviselli A, Sica V, Petrini L, Grasso L, et al. Therapy of Graves' disease with sodium ipodate is associated with a high recurrence rate of hyperthyroidism. J Endocrinol Invest. 1991;14(10):847-851.

20. Azizi F. Environmental iodine intake affects the response to methimazole in patients with diffuse toxic goiter. J Clin Endocrinol Metab. 1985;61(2):374-377.

21. Kasai K, Suzuki H, Shimoda SI. Effects of propylthiouracil and relatively small doses of iodide on early phase treatment of hyperthyroidism. Acta Endocrinol (Copenh). 1980;93(3):315-321.

22. Hiraiwa T, Ito M, Imagawa A, Takamatsu J, Kuma K,
Miyauchi A, Hanafusa T. Restriction of dietary Iodine does not ameliorate the early effect of anti-thyroid drug therapy for Graves' disease in an area of excessive iodine intake. J Endocrinol Invest. 2006;29(4):380-384.

23. Yabuta $T$, Ito $Y$, Hirokawa $M$, Fukushima $M$, Inoue $H$, Tomoda C, Higashiyama T, et al. Preoperative administration of excess iodide increases thyroid volume of patients with Graves' disease. Endocr J. 2009;56(3):371375.

24. Terry FD. Pathogenesis of Graves' disease. In: Lewis EB, David SC, eds. Werner and Ingbar's the thyroid. 10th ed. Philadelphia: Lippincott Williams \& Wilkins, 2012;356-369. 